\title{
Fulminant Emphysematous Pyelonephritis (EPN) in a Diabetic Patient Suspected of Having Multiple Tumors
}

\author{
Junko Sato ${ }^{1}$, Hajime M. Koyano ${ }^{1}$, Ruri Sasaki ${ }^{1}$, Yoshie Koike ${ }^{1}$, \\ Sosuke Sugimura ${ }^{2}$ and Hirotaka Watada ${ }^{3}$
}

\begin{abstract}
Multiple tumors in the liver, kidney, and on the posterior side of the urinary bladder were accidentally found when a diabetic woman visited the hospital. She refused to undergo surgery; therefore, she was only observed for 2 months. Subsequently, she was found unconscious at home and diagnosed with urinary infection, sepsis, and hyperglycemic hyperosmolar non-ketotic coma. The case followed a fulminant course, and she soon died. Postmortem computed tomography revealed emphysematous pyelonephritis (EPN), a rare, lifethreatening infection. This case highlights the importance of carefully managing infection in diabetic patients, and it may contribute to clarifying the pathogenesis of EPN.
\end{abstract}

Key words: emphysematous pyelonephritis, diabetes mellitus, hyperglycemic hyperosmolar non-ketotic coma, Klebsiella pneumoniae

(Inter Med 49: 2317-2320, 2010)

(DOI: 10.2169/internalmedicine.49.4000)

\section{Introduction}

Emphysematous pyelonephritis (EPN) is a rare but severe and acute necrotizing infection of the renal parenchyma and perirenal tissue, characterized by gas formation. The first case of EPN was documented in 1898 by Kelly and MacCullum (1). In Japan, the first case was reported in 1974, and 136 cases were reported by 2007 (2-5).

Uncontrolled diabetes mellitus is the main risk factor for EPN, and more than $80 \%$ of EPN cases are associated with diabetes mellitus (3). The mortality rate of EPN is $11-42 \%$. Percutaneous catheter drainage is usually necessary, failing which nephrectomy should be performed. Even though the mortality rate is high, the precise mechanism of EPN onset is debatable at the present time. We believe that the present case is a representative case of EPN and thus worthy to document its course from early development to the patient's sudden death.

\section{Case Report}

A 61-year-old woman visited our hospital for multiple tumors, which had been accidentally found at a neighboring hospital one-and-a-half months previously. The patient had been regularly visiting a doctor at the neighboring hospital for high blood pressure (from the age of 56) and diabetes mellitus (from the age of 46). The patient's history was as follows: she had had a cesarean delivery at the age of 28 . Her elder sister and younger brother both had diabetes mellitus. On admission, her height was $152 \mathrm{~cm}$; weight, 55.5 $\mathrm{kg}$; body temperature, $36.3^{\circ} \mathrm{C}$; blood pressure, $128 / 73$ $\mathrm{mmHg}$; and pulse, 60 beats per minute.

The tumors in the liver, right kidney, and on the posterior side of the urinary bladder were observed using enhanced computed tomography. A biopsy sample of the tumor on the posterior side of the urinary bladder was collected by performing laparoscopic surgery. However, this sample was found to contain only blood. We conducted a thorough examination of the patient (by using blood and urine examina-

\footnotetext{
${ }^{1}$ Division of Metabolism and Endocrinology, Department of Internal Medicine, Juntendo Urayasu Hospital, Urayasu, ${ }^{2}$ Department of Urology, Juntendo Urayasu Hospital, Urayasu and ${ }^{3}$ Division of Metabolism and Endocrinology, Department of Internal Medicine, Juntendo University, Tokyo

Received for publication May 25, 2010; Accepted for publication August 5, 2010

Correspondence to Dr. Junko Sato, jsato@juntendo.ac.jp
} 


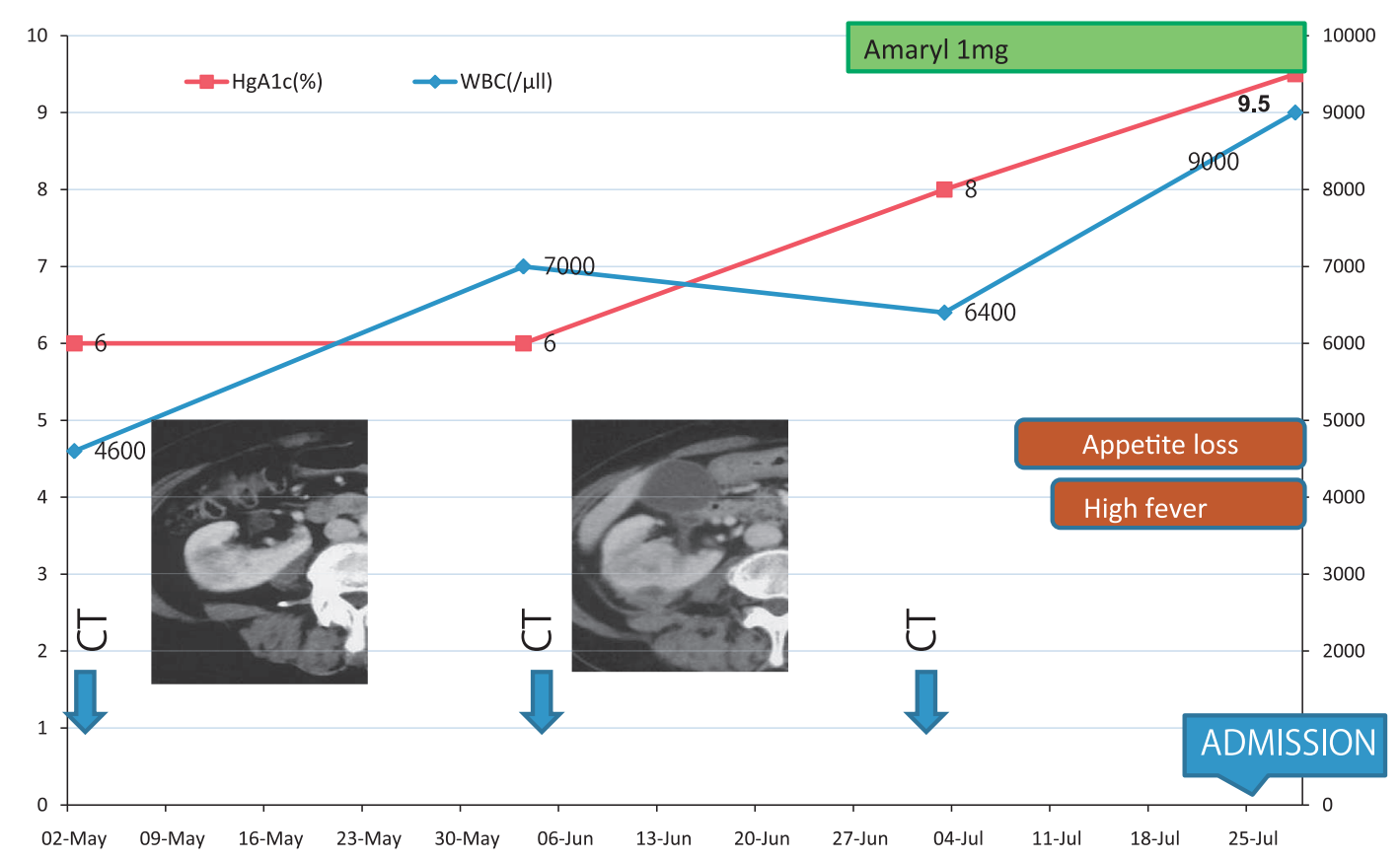

Figure 1. Clinical course.

tion, upper and lower endoscopy, and contrast radiography of the small intestine). There were no abnormal findings, except atrophic gastritis, slight anemia $(\mathrm{Hg}, 10.7 \mathrm{~g} / \mathrm{dL})$, and hyperglycemia after a meal (glucose, $149 \mathrm{mg} / \mathrm{dL}$ ). The patient did not have infections such as HIV, hepatitis B, hepatitis $\mathrm{C}$, or syphilis. The levels of all tumor markers were within the normal range (CEA, $1.7 \mathrm{ng} / \mathrm{mL}$; CA19-9, $6.4 \mathrm{U} /$ $\mathrm{mL}$; CA72-4, below detectable levels; and CA125, $6.4 \mathrm{U} /$ $\mathrm{mL})$. Her diabetes mellitus had been controlled without medication at the neighboring hospital.

The surgeon and the urologist recommended that the patient undergo celiotomy, which can be used to observe the tumors directly and precisely. However, the patient refused. Therefore, monthly observation with enhanced computed tomography was planned.

The first abdominal CT performed in early May revealed a cyst and several low-density areas in the liver (Fig. 1). In early June, the low-density areas of the liver became smaller; however, almost immediately, a new low-density area started to appear. This suggested that the low-density areas were actually abscesses in the liver. However, the WBC count, C-reactive protein level, and body temperature were within normal limits, that is, there were no symptoms of infection. Moreover, a low-density area of about $30 \mathrm{~mm}$ size was found in the right kidney in early May. In early June, several low-density areas were found in the kidney, and a tumor was also present on the posterior side of the right kidney. In early July, these low-density areas and the tumor had become enlarged (Fig. 2). Therefore, the urologist decided to perform nephrectomy of the right kidney. During this period, the patient's diabetes mellitus worsened, for which she was given glimepiride (Amaryl; $1 \mathrm{mg} /$ day).

In mid-July, the patient started experiencing appetite loss and fatigue at home. In spite of consuming proper meals, she had been drinking a large amount of carbonated soft drinks (soda pop, etc.). Within a week, her body temperature reached $39^{\circ} \mathrm{C}$ (as reported by her daughter), and she experienced much more thirst, for which she consumed more than $2 \mathrm{~L}$ of carbonated soft drinks per day without visiting the hospital. At the end of July, the patient was found in an unconscious state by her family at $2 \mathrm{AM}$. After being taken to the neighboring hospital, she was diagnosed with hyperglycemia $(1,062 \mathrm{mg} / \mathrm{dL})$, for which insulin was administered. After recovering from the respiratory arrest that occurred soon after she was taken to the hospital, the patient was transferred and admitted to our hospital at 10 : 50 AM.

The patient's status on admission was Japan Coma Scale, III-300; body temperature, $39.1^{\circ} \mathrm{C}$; blood pressure, 104/61 $\mathrm{mmHg}$; pulse, 107 beats per minute; $\mathrm{SpO}_{2}, 98 \%$ (8 L/min oxygen). ECG was normal, and the chest $\mathrm{X}$ ray (Fig. 3) suggested the existence of pneumonia. The laboratory data on admission were as follows: WBC count, 9,000/ $\mu \mathrm{L} ; \mathrm{Hg}$,

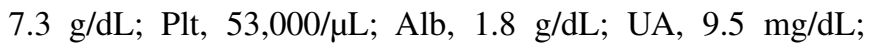
Ch-E, 1,925 IU/L; AST, 23 IU/L; ALT, 36 IU/L; BUN, 66 $\mathrm{mg} / \mathrm{dL} ; \mathrm{Cr}, 3.34 \mathrm{mg} / \mathrm{dL}$; Na, $142 \mathrm{mM} / \mathrm{L} ; \mathrm{K}, 4.3 \mathrm{mM} / \mathrm{L} ; \mathrm{Cl}$, 103 mM/L; Glu, 887 mg/dL; HbA1c, 9.5\%; CRP, 20.6 mg/ $\mathrm{dL}$; GAD antibody, negative; ketones, negative; $\mathrm{S}-\mathrm{C}$ peptide, $0.27 \mathrm{ng} / \mathrm{mL}$; S-osmolarity, $361 \mathrm{mOsm} / \mathrm{L} ; \mathrm{U}-\mathrm{WBC},(+++)$; and U-bacteria, (+++). Klebsiella pneumoniae was isolated from the blood and urine cultures.

On the basis of these findings, she was diagnosed with hyperglycemic hyperosmolar non-ketotic coma (HONK), urinary tract infection, and sepsis. Continuous subcutaneous insulin injection was initiated. Meropenem, gammaglobulin, and gabexate mesilate were also administered and red blood cells and blood platelets were transfused. However, the patient's blood pressure started to decrease at $2 \mathrm{PM}$, and she was declared to be dead at $7: 21 \mathrm{PM}$. 


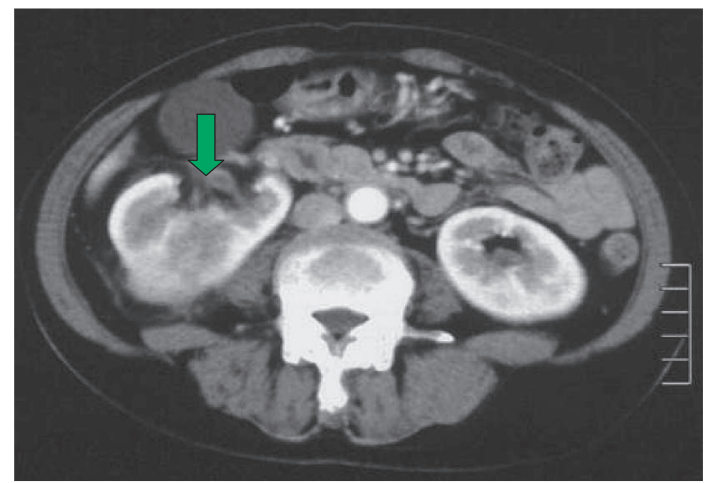

Figure 2. Computed tomography revealed enlarged lowdensity areas and a tumor in the right kidney (arrow); therefore, the urologist decided to perform a nephrectomy of the right kidney.

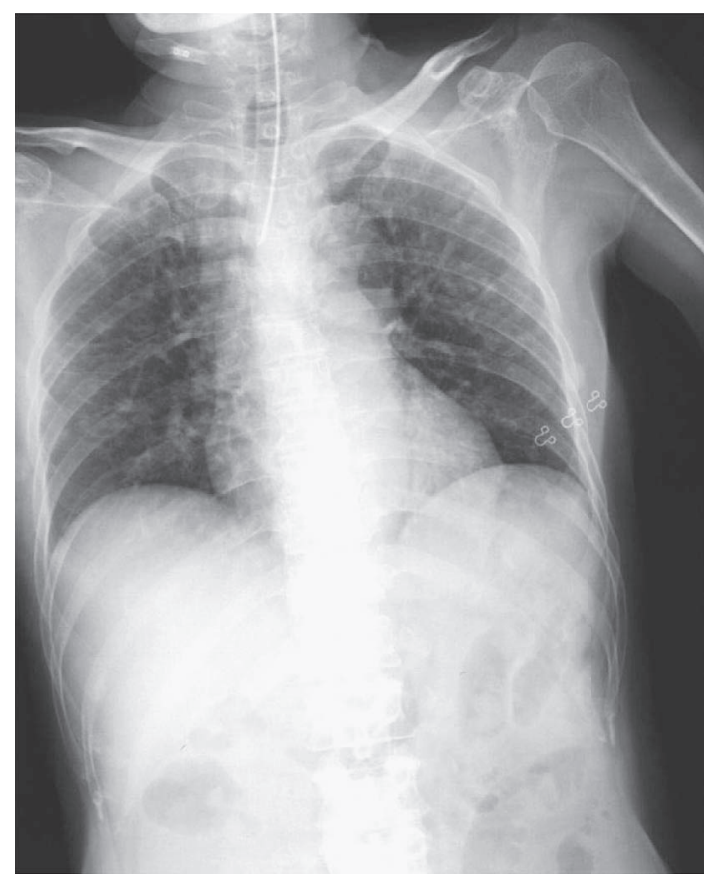

Figure 3. Chest $\mathrm{X}$ ray on admission suggested the existence of pneumonia.

Since the course of the disease was fulminant, we suggested that an autopsy be performed. However, the patient's family only approved computed tomography taken one hour after her death, which revealed that the right kidney was emphysematous, and the gas extended beyond the Gerota fascia of the left kidney (Fig. 4). Pneumonia, liver abscesses, peritonitis, slight brain atrophy, and sinusitis were confirmed using the tomography scan. Finally, we diagnosed the patient with fulminant EPN.

\section{Discussion}

During the early stages of the 61-year-old woman's hospitalization, the formation of abscesses, which grew slowly, was probably observed; however, the WBC count, C-reactive

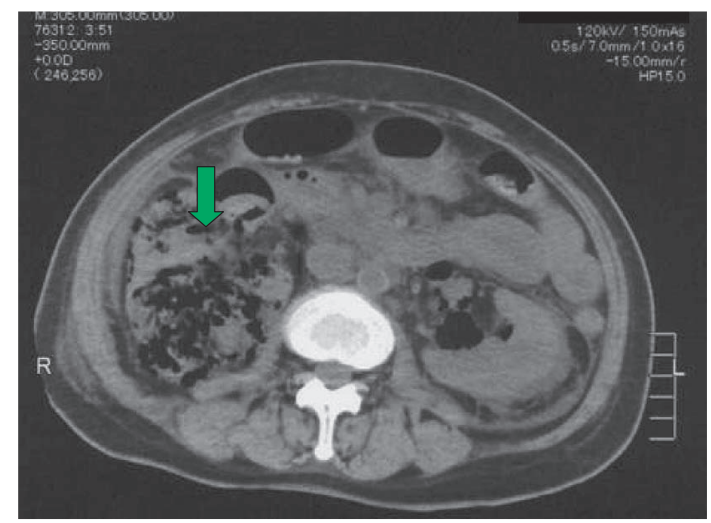

Figure 4. Computed tomography conducted postmortem showed that the right kidney (arrow) was emphysematous, and the gas extended beyond the Gerota fascia of the left kidney.

protein level, and body temperature were within the normal limits, and there were no symptoms of infection. The only abnormal finding was that the patient's anemia had been increasing gradually.

Four factors have been postulated to be involved in the pathogenesis of EPN: infection by gas-forming bacteria, high levels of tissue glucose, impairment in tissue perfusion, and defects in the immune response (such as those observed in patients with diabetes mellitus) (6-12). In our patient, $K$. pneumoniae, a gas-forming bacterium, was isolated from the blood and urine cultures. Escherichia coli and K. pneumoniae are the most common pathogens causing EPN $(11,13)$. On the day of admission, the patient's blood glucose level was more than $1,000 \mathrm{mg} / \mathrm{dL}$, which encouraged anaerobic fermentation, and the presence of abscesses resulted in impaired tissue perfusion. Further, she had a defective immune response because of uncontrolled diabetes mellitus. The HbA1c level was $9.5 \%(\mathrm{Hg}, 7.3 \mathrm{~g} / \mathrm{dL})$ on the day of admission.

This infection caused by the formation of multiple abscesses and the increased consumption of carbonated soft drinks (PET bottle syndrome) deteriorated the diabetes mellitus, and finally led to HONK. EPN and HONK together worsened the condition and led to sudden death.

Usually, the most useful tool for diagnosing EPN is an abdominal CT scan (13). According to the scan findings, Jeng-Jong $\mathrm{H}$. et al divided EPN into 4 classes: class 1, gas in the collecting system only (known as emphysematous pyelitis); class 2, gas in the renal parenchyma but not in the extrarenal space; class $3 \mathrm{~A}$, presence of gas or abscess in the perinephric space; class $3 \mathrm{~B}$, presence of gas or abscess in the pararenal space; and class 4 , bilateral or unilateral EPN. In a case of class $4 \mathrm{EPN}$, percutaneous catheter drainage is necessary, failing which nephrectomy should be performed (13-15). However, we could not treat the present patient in this way because of the fulminant EPN.

This is a rare case showing the progression of EPN from early development to the patient's sudden death. Patients 
with poorly controlled diabetes mellitus should be carefully monitored for the development of EPN. Further case studies are also required to clarify the pathogenesis of EPN.

\section{References}

1. Kelly HA, MacCallum WG. Pneumaturia. JAMA 31: 375-381, 1898.

2. Masago T, Watanabe $\mathrm{T}$, Isoyama $\mathrm{T}$, et al. Experiences of emphysematous pyelonephritis: 2 cases. Hinyouki Geka (Japanese Journal of Urological Surgery) 20: 1323-1326, 2007 (in Japanese, Abstract in English).

3. Kozakai N, Oya M, Kuroda K, Murai M. A survival case of emphysematous pyelonephritis accompanied by septic shock and disseminated intravascular coagulation (DIC). Hinyouki Geka (Japanese Journal of Urological Surgery) 18: 67-70, 2005 (in Japanese, Abstract in English).

4. Kumagai S, Muraki O, Date T. Emphysematous pyelonephritis treated with conservative therapy; indication of surgical therapy. Hinyouki Geka (Japanese Journal of Urological Surgery) 19: 1131-1134, 2006 (in Japanese, Abstract in English).

5. Takahashi K, Matsumoto T. Limitations of conservative treatment and indications of severe renal infection. Nihon Kagaku Ryouhoukai Zasshi 51: 439-446, 2003 (in Japanese).

6. Falagas ME, Alexiou VG, Giannopoulou KP, et al. Risk factors for mortality in patients with emphysematous pyelonephritis: a meta-analysis. J Urol 178: 880-885, 2007.
7. Nayeemuddin M, Wiseman OJ, Turner AG. Emphysematous pyelonephritis. Nat Clin Pract Urol 2: 108-112, 2005.

8. Tseng CC, Wu JJ, Wang MC, Hor LI, Ko YH, Huang JJ. Host and bacterial virulence factors predisposing to emphysematous pyelonephritis. Am J Kidney Dis 46: 432-439, 2005.

9. Huang JJ, Chen KW, Ruaan MK. Mixed acid fermentation of glucose as a mechanism of emphysematous urinary tract infection. J Urol 146: 148-151, 1991.

10. Tang HJ, Li CM, Yen MY, et al. Clinical characteristics of emphysematous pyelonephritis. J Microbiol Immunol Infect 34: 125-130, 2001.

11. Teruya H, Mouri $T$, Kagawa $H$, et al. Emphysematous pyelonephritis successfully treated by early intervention using a renoureteral catheter. J Infect Chemother 15: 195-198, 2009.

12. Horino T, Onodera S. Emphysematous pyelonephritis, xanthogranulomatous pyelonephritis. Hinyouki Geka (Japanese Journal of Urological Surgery) 21: 447-451, 2008 (in Japanese).

13. Jeng-Jong H, Chin-Chung T. Emphysematous pyelonephritis clinicoradiological classification, management, prognosis, and pathogenesis. Arch Intern Med 160: 797-805, 2000.

14. Vollans SR, Sehjal R, Forster JA, Rogawski KM. Emphysematous pyelonephritis in type 2 diabetes: A case report of an undiagnosed ureteric colic. Cases Journal 1: 192, 2008.

15. Abdu-Halim H, Kehinde EO, Abdeen S, et al. Severe emphysematous pyelonephritis in diabetic diagnosis and aspects of surgical management. Urol Int 75: 123-128, 2005.

(C) 2010 The Japanese Society of Internal Medicine http://www.naika.or.jp/imindex.html 